\title{
Vagotomy by chemoneurolysis: an experimental study in the rat
}

\author{
T V TAYLOR.* S HOLT, P L R ANDREWS, AND R C HEADING
}

From Manchester Roval Infirmary, Manchester; University of Alberta, Canada; University of Edinburgh; and Edinburgh Roval Infirmary, Edinburgh

SUMMAR' In the pylorus ligated rat vagotomy has been attempted by chemoneurolysis with alcohol. Surgical truncal vagotomy reduced the intragastric acid produced in a five-hour period from $31(0 \pm 17 \cdot 6$ (SD) $\mu \mathrm{mol}$ to $5 \cdot 4 \pm 2.4 \mu \mathrm{mol}$ with a marked reduction in the ulcer score. Injection of $30 \%$ alcohol in the region of the vagal trunks at the level of the lower oesophagus produced a marked reduction in gastric acid secretion $(7 \cdot 6 \pm 3.7 \mu \mathrm{mol})$. Injection of solutions of alcohol in concentrations over $10 \%$ along the lesser curvature reduced gastric acid output to less than 10 $\mu \mathrm{mol}$ with a corresponding reduction in the ulcer score. Four months after the injection of $30 \%$ alcohol along the lesser curvature the acid output was $23 \cdot() \pm 5 \cdot 7 \mu \mathrm{mol}$ and no gastric ulceration occurred.

Lumbar sympathectomy performed by percutaneous injection of phenol or alcohol was introduced by Haxton' in 1949 and is now routinely used in many centres. Deloyers et al studied the selective destruction of the myenteric nerve plexus of the lower oesophagus by submucosal injection of 5'c phenol: in this study phenol was shown to be a specific destructive agent for nervous tissue and at 5 "'c concentration produced minimal fibrosis and no necrosis. Schapiro and Britt ${ }^{3}$ reported that the introduction of phenol into the submucosa of denervated canine fundic gastric pouches produced a marked reduction in secretory responses to histamine and food. These workers suggested that selective destruction of Meissner's submucosal plexus had occurred without reduction in the number of parietal cells or changes in histological appearance of the gastric tissues. In view of the possible toxic effects of phenol the use of ethyl alcohol as an alternative neurotoxin was investigated by Schapiro et $\mathrm{al}^{+}$Marked secretory inhibition of histamine and food stimulated gastric secretion was produced in fundic pouches of dogs after submucosal injections of alcohol. The purpose of this study was to investigate the feasibility of damaging the vagus nerve by means of the injection

\footnotetext{
* Address for reprints: Mr T V Taylor. Manchester Royal Intirmary. Oxford Road. Manchester M113 4WL

Received for publication + Ma! 1982
}

of various concentrations of alcohol in the region of the intra-abdominal vagus nerves of the rat.

ANATOMICAI. CONSIDERATIONS

The vagus nerves in the rat bear a similar anatomical relationship to the stomach to that in man. The anterior and posterior vagus nerve trunks continue along the lesser curvature of the stomach as the nerves of Latarjet. Branches leaving these nerves are closely applied to the serosa of the anterior and posterior gastric walls before penetrating the muscularis to innervate the mucosal syncitium of nerves.

\section{Methods}

\section{MATERIALS}

Ten groups of eight Sprague-Dawley rats weighing between $250-350 \mathrm{~g}$ were fasted from solid food for 24 hours before being anaesthetised with ether (Table 1). In a control group of rats, group 1 , pylorus ligation alone was performed. ${ }^{5}$ Group 2 underwent truncal vagotomy, performed by dividing the vagus nerve trunks as they run along the lower oesophagus, in addition to pylorus ligation. In group $3,0.5 \mathrm{ml} 30 \%$ alcohol was injected around the vagus nerve trunks at the level of the lower oesophagus and pylorus ligation was performed. In groups 4-8 an attempt was made to perform highly selective vagotomy by injection of alcohol along the lesser curvature of the stomach, from the oesophago- 
Table 1 Groups of eight Sprague-Dawley rats fasted for 24 hours and anaesthetised

\begin{tabular}{cl}
\hline Group & \\
\hline 1 & Pyloric ligation (PL) \\
2 & Truncal vagatomy + PL \\
3 & $30 \%$ alcohol around the lower oesophagus + PL \\
4 & $10 \%$ alcohol lesser curve + PL \\
5 & $25 \%$ alcohol lesser curve + PL \\
6 & $30 \%$ alcohol lesser curve + PL \\
7 & $5(1 \%$ alcohol lesser curve + PL \\
8 & $10 \% \%$ alcohol lesser curve + PL \\
9 & $20 \%$ ammonium sulphate \\
10 & $30 \%$ alcohol - delay four months - PL \\
\hline
\end{tabular}

gastric junction to the incisura, using a fine-bore needle. Concentrations of alcohol varying between 10 and $100 \%$ were used. In group 9 a $20 \%$ solution of ammonium sulphate was used as the neurotoxin and injected along the lesser curvature. After ligating the pylorus in all groups the abdomen was closed and anaesthesia was maintained by intraperitoneal pentobarbitone (Sagital). The animals were placed in an incubator in which their body temperature was maintained and they were killed after five hours, this was the period of time during which stable anaesthesia could be reliably achieved. The stomach was removed and the gastric contents were collected for estimation of $\mathrm{pH}$, volume, and acid content. The number of ulcers or erosions was then counted and after photography samples of the excised stomachs were examined histologically by light microscopy. In group 10 a solution of $30 \%$ alcohol was injected along the lesser curvature of the stomach, these animals were allowed to recover and four months later at a second laparotomy pylorus ligation was carried out before they were killed, five hours later. The longer term effects of the injection of concentrations of 75 and $100 \%$ alcohol along the lesser curvature were also investigated. In addition, a group of eight anaesthetised rats with sham operation but no pyloric ligation were placed in the incubator for five hours.

\section{Results}

The rats in which pyloric ligation alone was performed (group 1) had a mean acid output of $310 \pm 17 \cdot 6$ (SD) $\mu \mathrm{mol}$ and marked ulceration occurred in the stomachs of each of these animals. The total ulcer score in this group of eight rats including erosions was 248 (Table 2). Truncal vagotomy, performed surgically (group 2), markedly reduced both acid output, to a level of $5 \cdot 2 \pm 2.4 \mu \mathrm{mol}$, and the tendency to ulcerate, the
Table 2 Ulcer score in pvloric ligated rats

\begin{tabular}{lllr}
\hline Group & Description & $\begin{array}{l}\text { No of } \\
\text { rats }\end{array}$ & $\begin{array}{l}\text { No of } \\
\text { ulcers }\end{array}$ \\
\hline 1 & Pyloric ligation alone & 8 & 248 \\
2 & TV + PL & 8 & 6 \\
3 & TV with alcohol + PL & 8 & 12 \\
4 & $10 \%$ alcohol lesser curve + PL & 8 & 33 \\
5 & $25 \%$ alcohol lesser curve + PL & 8 & 25 \\
6 & $30 \%$ alcohol lesser curve + PL & 8 & 28 \\
7 & $50 \%$ alcohol lesser curve + PL & 8 & 5 \\
8 & $100 \%$ alcohol lesser curve + PL & 8 & 5 \\
9 & $30 \%$ alcohol-delay four months & 8 & 0 \\
\hline
\end{tabular}

ulcer score being six. The injection of $30 \%$ alcohol around the vagal trunks (group 3) produced a similar degree of secretory inhibition and reduction of mucosal ulceration compared with truncal vagotomy. When $10 \%$ alcohol was injected along the lesser curvature of the stomach the acid output was $7 \cdot 6 \pm 5 \cdot 1 \mu \mathrm{mol}$ and $30 \%$ alcohol produced an output of $3.8 \pm 1.9 \mu \mathrm{mol}$. With concentrations of 50 and $100 \%$ alcohol no further significant reduction in acid output was achieved (groups 4-8). The tendency to ulcerate was markedly reduced with all concentrations of alcohol and this, in conjunction with the magnitude of acid secretory inhibition, suggested that each injection dose was capable of producing interruption of the functional vagal innervation, at least temporarily. Ammonium sulphate in a concentration of $20 \%$ when injected along the lesser curvature failed to produce significant inhibition of acid secretion (Fig. 1).

The group that was left for four months (group

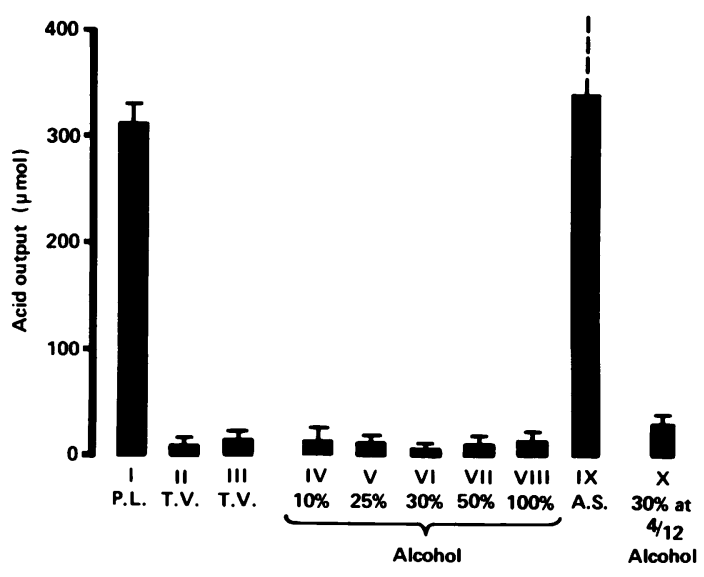

Figure Acid output ( $\mu \mathrm{mol})$ in the groups of rats studied 
10) after a preterminal pylorus ligation study had an acid output of $23.0 \pm 5 \cdot 7 \mu \mathrm{mol}$ and the ulcer score in these animals was zero.

A group of eight animals was also observed after the injection of $100 \%$ alcohol along the lesser curvature; four of these animals developed gross gastric dilatation and necrosis of the lesser curve with leakage of gastric contents and fatal peritonitis. In a group injected with $75 \%$ alcohol there were two deaths from this cause. Several of the group injected with $30 \%$ alcohol and studied four months postinjection had grossly dilated stomachs at laparotomy and, despite fasting for 24 hours, these contained considerable gastric residue; it was assumed that the innervation of the antrum in addition to the parietal cell area had been damaged by the alcohol and a situation similar to surgically induced truncal or selective vagotomy had resulted. Histological examination of the lesser curvature of the stomach in those rats in which $30 \%$ alcohol had been injected four months previously revealed no abnormality in the cellular architecture.

After five hours in the incubator under general anaesthesia the sham operated group did not produce ulcers, and after being killed there was little or no resting juice in the stomach.

\section{Discussion}

This study performed in the rat showed that alcohol in concentrations of over $10 \%$ when applied to the vagus nerve produced functional damage with little damage to other tissues when concentrations of up to $50 \%$ were used. After four months acid output remained markedly reduced when a concentration of $30 \%$ was used. Absolute alcohol in the rat may produce extensive tissue damage with necrosis of the gastric wall.

The period of time between ligating the pylorus and killing the animal (five hours) was less than that described by Shay ${ }^{5}$ (16 hours). The reason for this was that, in order to comply with Home Office regulations, the animals were kept fully anaesthetised after ligating the pylorus.

Despite placing the rats in an incubator we were able to reliably maintain anaesthesia for only a minimum of about six hours before some of the animals died, but all survived for five hours. Although the period of time was reduced, all of the control animals with pyloric ligation developed ulceration and gastric erosions, and secreted acid in sufficient quantities for this to be accurately measured.

Conversely, animals placed in the incubator after sham operation did not ulcerate. It is apparent from this study that the concentrations of alcohol used were capable of penetrating and damaging the fine vagus trunks in the rat.

Although the conditions of this study are far from physiological, use has been made of a model (the pyloric ligated rat) which produces a marked acid secretory response with, in the presence of an intact vagus, an inevitable tendency to ulcerate. ${ }^{6}$ After vagotomy this propensity for ulceration is markedly reduced. 56

The study indicates that the application of alcohol in the region of the vagus in the rat is capable of reducing acid output and the number of experimentally induced ulcers. In man the fibrous tissue Surrounding the vagal trunks or the thickness of the trunks themselves would probably prevent penetration by alcohol.

The authors are grateful to Pyser Ltd for financial support.

\section{References}

1 Haxton HA. Chemical sympathectomy. Br Med J 1969; 1: $1026-8$

2 Deloyers L, Cordier R, Duprez MA. A new approach to the so-called physiology of cardiospasm; experimental production of cardiospasm in cats after destruction of Auerbach's plexus. Ann Surg 1957; 146: $167-71$.

3 Schapiro H, Britt LG. Injection of 5\% phenol into submucosa of denervated gastric pouch. Arch Surg 1967; 94: 841-5.

4 Schapiro H, McDougal HD, Carwell G, Rosato FE, Jackson NH. Submucosal vagotomy of the canine gastric fundus. Surg Gynec Obstet 1977; 144: 534-6.

5 Shay H, Komarov SA, Gruenstein M. Effects of vagotomy in the rat. Arch Surg 1949; 59: 210-26.

6 Hakanson R. Hedenbro J, Leidberg G. Sundler F. Vallgren S. Mechanisms of gastric acid secretion after pylorus and ocsophagus ligation in the rat. J Physiol 198(); 3105: 139-45. 